\title{
Controlling the Hypnotic Drug (propofol) to maintain a stable depth of Anesthesia, in Dogs
}

\author{
Susana Brás \\ IEETA \\ Universidade de \\ Aveiro \\ Aveiro, Portugal \\ susana.bras@ua.pt
}

\author{
Lénio Ribeiro \\ Hospital Veterinário \\ do Porto \\ Porto, Portugal \\ lenioribeirovet@gmail \\ .com
}

\author{
David A. Ferreira \\ CBIOS \\ ULHT \\ Lisboa, Portugal \\ ferreiradav@gmail.co \\ $\mathrm{m}$
}

\author{
Luis Antunes \\ CITAB, CVD \\ UTAD \\ and \\ Laboratory Animal \\ Science, IBMC \\ Portugal \\ lantunes@utad.pt
}

\author{
Catarina S Nunes \\ DCeT \\ Universidade Aberta \\ Lisboa, Portugal \\ CatarinaS.Nunes@uab \\ .pt
}

\begin{abstract}
The development of control and automatic systems is important to guarantee a stable anesthesia, with no under or over dosage, and no awareness episodes. In this study a controller for the Cerebral State Index (CSI - an electroencephalogram derived signal) was developed. This study was a simulation study, the CSI was modeled using a fuzzy logic model with two inputs the effect-site concentration of propofol and the electromyography (EMG). The controller was tested using constant and variable references in an exhaustive set of simulations. The controller developed presents a good performance in all simulations and the controlled variable seems to be influenced by electromyography level. A controller for propofol anesthesia for veterinary use is an important step towards the improvement of animal welfare. The overall aim is to improve animal safety and comfort.
\end{abstract}

Keywords-control systems; anaesthesia; dogs; cerebral state index; fuzzy logic

\section{INTRODUCTION}

General anesthesia can be defined as the lack of response and recall to noxious stimuli involving the use of three drugs: a muscle relaxant, an anesthetic (hypnotic) and an analgesic. A stable anesthesia is balanced between these components to guarantee the absence of any sensation or perception during a surgical intervention. The patient stability is very important.

Animal anesthesia is now presenting small advances. In most of the cases, the veterinarian surgeon is also anesthetist. Advisory and automatic systems to induce and maintain anesthesia will be a great development and help in veterinary anesthesia, improving animal welfare.

Anesthesia administered by a feedback control system of patients' clinical state will improve quality and safety, resulting in a better control of anesthesia and faster recovery [1]. The cerebral activity during anesthesia is depressed by hypnotic action. To guarantee a complete absence of any perception the cerebral activity should be continuously evaluated and monitored. This activity could be evaluated by different signals, the electroencephalogram (EEG), or signals derived from EEG like Bispectral Index (BIS - by Covidien), Cerebral State Index (CSI - by Danmeter), Entropy (by General
Electric), etc. All of these monitors evaluate unconsciousness, and proved to be very useful in monitoring depth of anesthesia in humans [2-4]. These derived signals are friendlier to understand, the EEG is a chaotic signal, difficult to understand. In the operating theater a rapid solution is needed most of the times. The derived EEG signals give a number inside a predefined interval, where different values represent different anesthesia stages.

In human medicine the most used depth of anesthesia monitor is the BIS. The BIS is composed from different measures of EEG using signal processing techniques including bispectral analysis, power spectral analysis, time domain analysis and also includes a human database comparison. In animals CSI had a good response to induction [5] [6], and it is now studied like an unconsciousness monitor for dogs. The CSI is an open system index calculation with multiparametric EEG processing based on fuzzy logic. These monitors differ in the index calculation method, but with the same interpretation.

In the present study, the CSI is used as a measure of unconsciousness in dog anesthesia. The numerical scale of CSI varies between "0" and "100", where values near 100 correspond to a brain electric activity of an "awake" brain, while a CSI of "0" indicates complete absence of brain electric activity, i.e., an isoelectric EEG line. CSI in dogs shows a correlation between increased anesthetic depth and lower CSI values due to decreased brain electric activity [5] [7].

The hypnotic level is achieved after the administration of hypnotic drugs, the anesthetics, in this case propofol, by Target Controlled Infusion (TCI). TCI is an anesthetic delivery system now widely used in human anesthesia [8]. The administration of propofol, by TCI uses as targets the plasma or effect-site concentrations, estimated by a three compartmental pharmacokinetic/pharmacodynamic mathematic models [9]. The plasma concentration is the amount of drug in a $m l$ unit of blood, the effect-site concentration is the drug concentration related to the effect in the target organ. In the case of hypnotics, the target organ is the brain. The pharmacokinetic model used, to describe propofol plasma concentration in dog, was developed by Beths [10], the pharmacodynamic variable $k_{e 0}$ was calculated by us [11]. The effect-site concentration is 
the amount of drug that produces real effect, providing CSI changes. In veterinary medicine, some reports have been published using TCI in dogs during induction and maintenance of anesthesia [10], [12], stating that TCI is an appropriate technique to be used in dogs. The TCI can improve the delivery rate to a desire clinical effect while minimizing side effects [13] and allows a more effective and easy control of depth of anesthesia [10].

The aim of this study was to develop a control system to control the effect-site concentration of propofol to obtain a stable level of unconsciousness in dog evaluated by CSI. The electromyography (EMG) proved to be very related with CSI signal, to achieve a stable CSI a stable EMG level is required. The CSI is the controlled variable. This study is a simulation study using a model developed with the clinical data o 14 dog surgeries. The CSI model was a fuzzy logic model, with two inputs the effect-site concentration of propofol and the EMG. The developed controller was a Proportional Integral Derivative (PID) controller. In the future, this system could be (after further tests) implemented in clinical use to help the veterinarian in his usual practice. The pharmacokinetics and pharmacodynamics are presented in section II, the relation between EMG and CSI is explained in section III, the fuzzy logic model of CSI is explained in section IV. The proportional integral derivative controller is presented in section V. Section VI presents the results. The conclusions are presented in section VII.

\section{PHARMACOKINETICS AND PHARMACODYNAMICS} (PK/PD)

PK/PD models simulate what the body does to the drug (pharmacokinetic) and what the drug does to the body (pharmacodynamic). The pharmacokinetic (PK) model used to describe the propofol plasma concentration in dogs was described by Beths [10]. It is a three-compartmental model describing the relation between the drug infusion rate and the drug plasma concentration. The plasma concentration is defined as the concentration in the central compartment (the

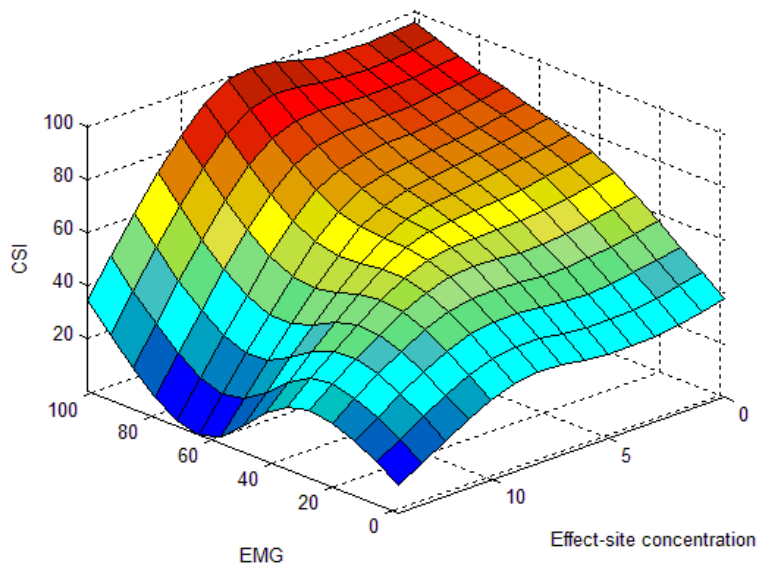

Fig. 1. Output surface of the fuzzy logic structure that describes CSI knowing effect-site concentration and EMG. blood). After the propofol administration to a central compartment, it is rapidly distributed to a second compartment, compose mainly by the muscular tissues, and slowly distributed to a third compartment, which corresponds mainly to the body fat tissue storage [9]. The pharmacodynamics describes the amount of drug that passes from central compartment to the effect organ (the brain) [11].

\section{EMG AND CSI}

The CSM monitor provides different measures. The Cerebral state index (CSI) is related with unconsciousness, electromyography (EMG) is related with muscular activity (EMG is given as a percentage, the energy of the EMG level in the $75-85 \mathrm{~Hz}$ frequency band [13]), burst suppression rate (BSR) is related with the suppression of EEG (the detection of isoelectric pattern), and signal quality index (SQI) related with the CSI signal quality. The CSI, EMG and BSR were related between them, an increase in BSR implies a decrease in CSI and EMG. But the strongest correlation was between EMG and CSI (a correlation coefficient of $0.68, p<0.001$ ).

The EMG influences CSI, the higher frequencies in EMG are noise in CSI, but the low frequencies are followed by alterations in CSI pattern.

\section{FUZZY LOGIC MODEL OF CSI}

This is a simulation study; the CSI (measure of unconsciousness) was modeled using fuzzy logic techniques. The effect-site concentration and the EMG are two variables that are directly related with CSI. The model was developed using 14 dogs, data were collected during routine surgeries. All dogs were subject to general anesthesia, induced and maintained using propofol. The dogs studied were between $18 \mathrm{~kg}$ and $35 \mathrm{~kg}(25.3 \pm 5.3 \mathrm{~kg})$ undefined pedigree housed at Hospital Veterinário do Porto under standard conditions.

The CSI model was a fuzzy logic model, the use of these type of models was related with the fact that the system is extremely nonlinear, there exists a lot of external interference, and there it is not possible determine a pattern. Fuzzy logic systems can relate variations of inputs to a certain value of output based on precise theory. The nonlinear character of fuzzy logic may contribute to a higher robustness of systems [14].

The model for CSI was developed using Takagi-SugenoKang theory. The fuzzy logic output surface is presented in Fig. 1.

\section{PROPORTIONAL INTEGRAL DERIVATIVE CONTROLLER (PID)}

PID controllers are the standard tool for industrial automation. The flexibility of the controller makes it possible to use PID control in many situations [15]. The continuous representation of PID controller is represented by (1).

$$
\dot{u}(t)=k\left(\dot{e}(t)+\frac{1}{T_{I}} e(t)+T_{D} \ddot{e}(t)\right)
$$




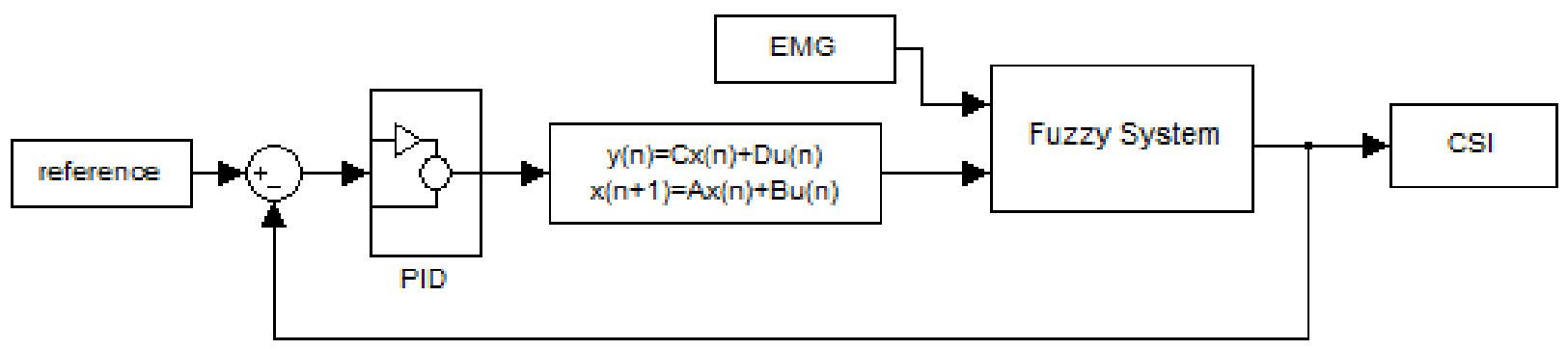

Fig. 2. System scheme of the controller.

The PID controller controls the CSI, regulating the effectsite concentration. EMG was only a variable that was strongly related with CSI (read only variable) (Fig. 2). The input of the controller was the error defined as error $=$ reference modelresponse, the output was the amount of drug necessary to obtain the desired reference.

\section{A. Ziegler-Nichols method}

The method used to tune the controller parameters was the Ziegler-Nichols method. Fig. 3 represents the response to a unit step response of a typical process. The gains of the PID controller was tunned using the parameters $a$ and $L$, represented in the figure. To apply this method it was needed that $0.1<L / T<0.6$ was verified. The regulator parameters are given in Table I [15].

\section{B. Performance Error}

The performance error was calculated according to (2). The inaccuracy (median absolute performance error) was calculated by (3), where $N_{i}$ is the number of values $P E$ obtained for the $i$ th subject [1].

$$
\begin{aligned}
& P E=\frac{(\text { measuredvalue }- \text { targetvalue })}{\text { targetvalue }} \times 100 \\
& M D A P E_{i}=\text { median }\left\{|P E|_{i j}, j=1, \ldots, N_{i}\right\}
\end{aligned}
$$

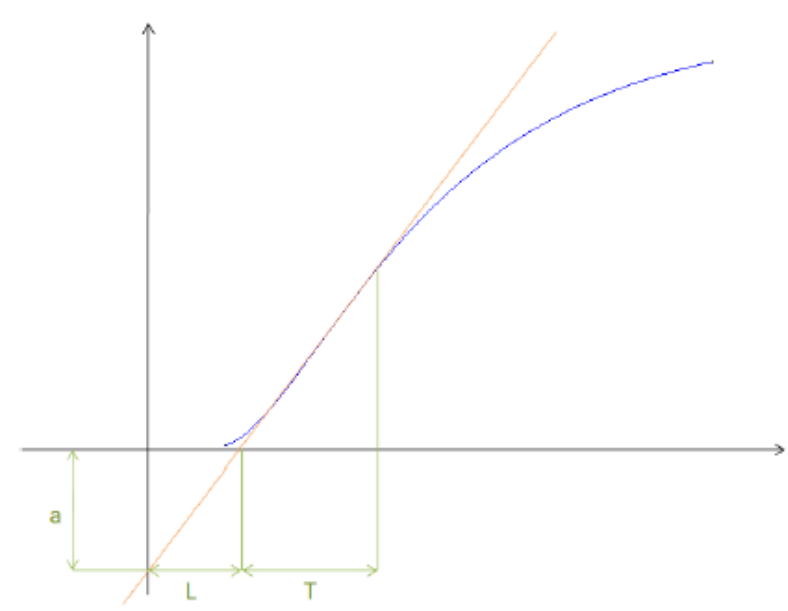

Fig. 3. Unit step response of a typical industrial process.
The performance of the developed controller will be tested using three different constant CSI references referring to three different anesthetic stages (deep anesthesia, anesthesia and sedation). The EMG has a big influence in the CSI model, so the controller will be tested using different EMG behaviors. In the initial test the EMG will be constant and related with CSI reference, in a second approach the EMG will be fixed at 10 and finally the EMG will be variable and increased in steps of 10 between 10 to 100 .

\section{RESULTS}

The controller should be robust, simple and precise. This controller has as input the error (deviation of the model output from the reference) and the output was the amount of drug necessary to obtain the desired reference. The developed controller was tested, with different CSI reference profiles, that were related to different anesthesia stages (CSI $=30$ - deeper anesthesia, CSI $=50$ - anesthesia, CSI $=70$ - sedation). In Table II it is presented the performance errors of the controller, simulating a dog of $18 \mathrm{~kg}$, and an input EMG adequate to the CSI reference. Fig. 4 shows the response of the controller to the constant references.

The response of the controller was influenced by the EMG tendency. If the EMG level was stable, the controller will be stable, as showed in Fig. 5. With the increase of the CSI
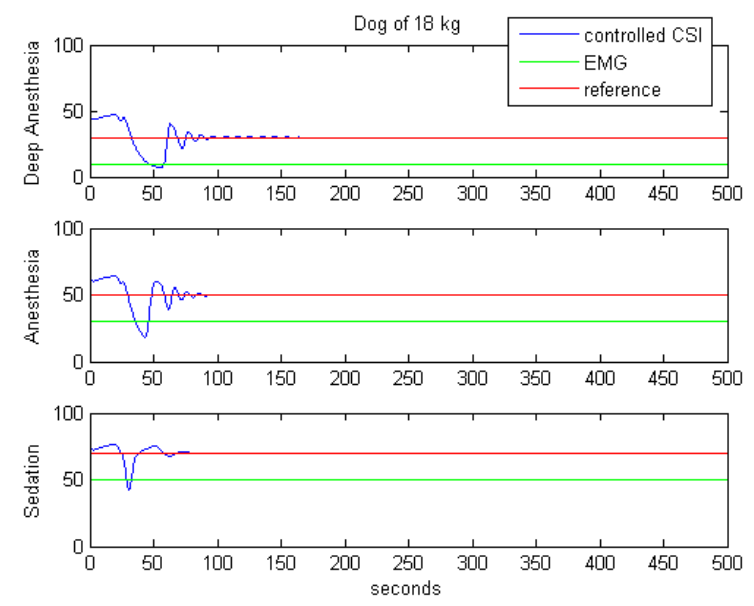

Fig. 4. Performance of the controller in three anesthetic stages, deep anesthesia $(\mathrm{CSI}=30)$, anesthesia $(\mathrm{CSI}=50)$ and sedation $(\mathrm{CSI}=70)$. 
reference, the performance of the controller, with an $\mathrm{EMG}=10$, was worst. The performance of the controller with a variable EMG level is shown in Fig. 6.

\section{CONCLUSIONS AND FUTURE WORK}

\section{A. Conclusions}

The aim of the study was to develop a controller that was able to achieve and maintain the CSI signal according to a reference. At this point only simulation studies were carried out, in the future as a new system to help in clinical practice and improve animal welfare. A good anesthesia is stable and balanced (the patient does not move, does not feel pain, is unconscious). Awareness during anesthesia worries anesthesiologists and patients alike. A balanced, controlled and stable anesthesia is desired by the patient and the anesthesiologist. The recovery of the patient should be fast and calm, so the amount of drug administered during surgery has to be controlled. This point has a cost impact, if the anesthesiologist could induce and maintain anesthesia with less drug, it is better for patient (less side effects, and fast recovery) and less money spent. An automatic control system maintains a certain level of unconsciousness, evaluating every time if a patient needs more drug.

In a simulation mode the developed controller showed a good performance, depending on the EMG values. The EMG has a high impact in the CSI model. If the input EMG was related with the CSI reference, the result of the controller would be good (Fig. 4). If the EMG was fixed at a constant level and the CSI reference was constant, but with different values (in this case in three different anesthetic stages), the performance of the controller was worst with the increasing of the CSI reference (Fig. 5). In a real case the EMG could vary along the surgery. The simulation with an EMG that varies in time shows that in the case of sedation the time until the
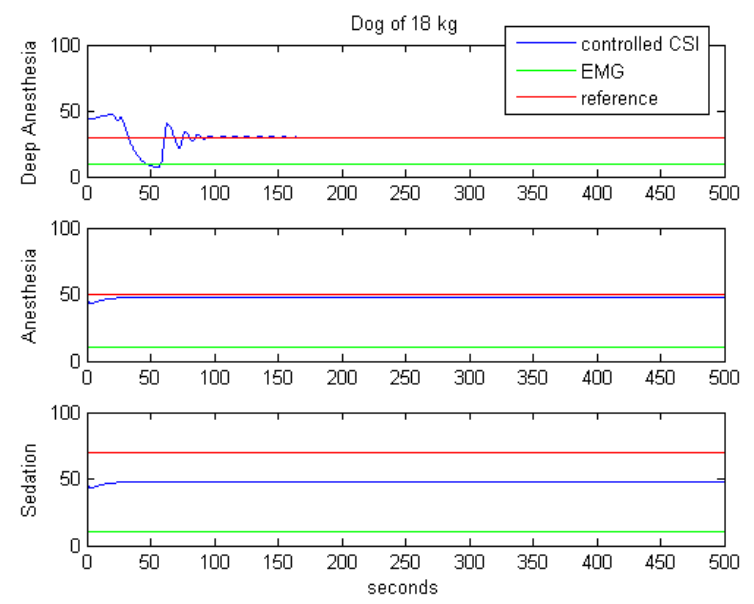

Fig. 5. Performance of the controller in three different references representing three anesthetic stages, deep anesthesia $($ CSI $=30$ ), anesthesia $(\mathrm{CSI}=50)$ and sedation $(\mathrm{CSI}=70)$. In this case the EMG was stable and equal to 10 in all three simulations.
TABLE I.

PARAMETERS OBTAINED BY THE ZIEGLER-NICHOLS STEP RESPONSE METHOD

\begin{tabular}{|l|l|c|c|}
\hline \multirow{2}{*}{$\begin{array}{c}\text { Controller } \\
\text { Type }\end{array}$} & \multicolumn{3}{|c|}{ Parameters } \\
\cline { 2 - 4 } & \multicolumn{1}{|c|}{$\boldsymbol{k}_{\boldsymbol{c}}$} & $\boldsymbol{T}_{\boldsymbol{i}}$ & $\boldsymbol{T}_{\boldsymbol{d}}$ \\
\hline $\mathrm{P}$ & $1 / \mathrm{a}$ & & \\
\hline $\mathrm{PI}$ & $0.9 / \mathrm{a}$ & $3 \mathrm{~L}$ & \\
\hline $\mathrm{PID}$ & $1.2 / \mathrm{a}$ & $2 \mathrm{~L}$ & $\mathrm{~L} / 2$ \\
\hline
\end{tabular}

TABLE II. MEDIAN ABSOLUTE PERFORMANCE ERROR (MDAPE) OF THE CONTROLLER IN THREE ANESTHETIC STAGES.

\begin{tabular}{|l|c|}
\hline \multicolumn{1}{|c|}{ Anesthetic stages } & MDAPE (\%) \\
\hline Deep Anesthesia & 0.0072 \\
\hline Anesthesia & 0.0037 \\
\hline Sedation & 0.0012 \\
\hline
\end{tabular}

controller achieves the desired anesthetic stage was longer. In the case of deep anesthesia, the controller achieves the target in a short time, but when the EMG suffers any alteration the controller has a short period of oscillation (Fig. 6).

A stable level of depth of anesthesia is important to guarantee good surgical conditions, dog's safety and fast recovery.

\section{B. Future Work}

The degree of sensitivity/resistance of the dog to the drugs has a great influence on the amount of drugs necessary during surgery, to maintain an adequate level of depth of anesthesia. Information extracted from models can be used to adapt the infusion rate of the drug, avoiding cases of over/under dosage.

The developed controller shows certain restrictions associated with EMG; in future the incorporation of a controller for EMG could be integrated in this system, to guarantee a stable and correct CSI controller. The most common dogs weight is about $18 \mathrm{~kg}$, but there are a lot of different dogs' weights, so future simulations will need to be done in order to observe the response of the controller to different dogs metabolisms (associated with dogs' weight). In a real surgery, at different time points of the surgery the unconsciousness stage of the patient was different so the CSI reference was not time constant, it changes with patient necessity, so in the future simulations with CSI variable references are required.

The developed controller could be incorporated in the operating theater helping veterinarian anesthesiologist. In the first phase of implementation it will have to be validated and tested, comparing the real and expected reactions of the dog. 

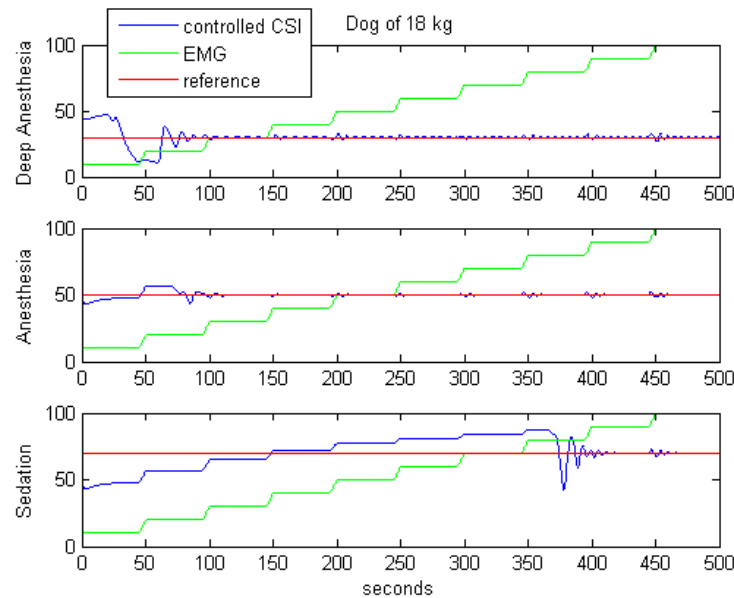

Fig. 6. Performance of the controller using three different references, deep anesthesia $(\mathrm{CSI}=30)$, anesthesia $(\mathrm{CSI}=50)$ and sedation $(\mathrm{CSI}=$ 70). In this case the EMG was variable, growing in steps of 10 .

\section{ACKNOWLEDGMENT}

C.S. Nunes would like to acknowledge the support of UISPA - System Integration and Process Automation Unit Part of the LAETA (Associated Laboratory of Energy, Transports and Aeronautics) an I\&D Unit of the Foundation for Science and Technology (FCT), Portugal. FCT support under projects: PEst-OE/EME/LA0022/2013, FCOMP-01-0124FEDER-022682 (ref. PEst-C/EEI/UI0127/2011), Incentivo/EEI/UI0127/2013 (Instituto de Engenharia Electrónica e Telemática de Aveiro, IEETA/UA, Aveiro, www.ieeta.pt). S. Brás acknowledges the postdoctoral grant by Cloud Thinking Project (ref. BPD/DETI/5259/2013), cofunded by QREN, "Mais Centro" program (CENTRO-07ST24-FEDER-002031).

\section{REFERENCES}

[1] T. Smet, M.M.R.F. Struys, S. Greenwald, E.P. Mortier, S.L. Shafer, "Estimation of Optimal Modeling Weights for a Bayesian-Based
Closed-Loop System for Propofol Administration Using the Bispectral Index as a Controlled Variable: A Simulation Study," Anesthesia \& Analgesia, vol. 105, pp. 1629-38, 2007.

[2] M. Iannuzzi, E. Iannuzzi, F. Rossi, L. Berrino, M. Chiefari, "Relationship between Bispectral Index, electroencephalographic state entropy and effect-site EC50 for propofol at different clinical endpoints," British Journal of Anaesthesia, vol. 94, pp 492-5, 2005.

[3] P. F. White, H. Ma, J. Tang, R. H. Wender, A. Sloninsky, R. Kariger, "Does the Use of Electroencephalographic Bispectral Index or Auditory Evoked Potential Index Monitoring Facilitate Recovery after Desflurane Anesthesia in the Ambulatory Setting?," Anesthesiology, vol. 100, pp 811-7, 2004.

[4] A. Ekman, L. Brudin, R. Sandin, "A Comparison of Bispectral Index and Rapidly Extracted Auditory Evoked Potentials Index Responses to Noxious Stimulation During Sevoflurane Anesthesia," Anesthesia Analgesia, vol 99, pp 1141-6, 2004.

[5] P. J. A. Bollen, H. Saxtorph, "Cerebral State Monitoring in Beagle dogs sedated with metedomidine," Veterinary Anaesthesia and Analgesia, vol 33, pp 237-240, 2006

[6] L. Ribeiro, D. Ferreira, N. Bressan, P. Amorim, L. Antunes, "Cerebral State Index (CSI) monitoring in dogs during induction of anaesthesia with propofol," European Journal of Anaesthesiology, vol. 24 (Suppl.39), 3AP8-11, 2007.

[7] L. M. Ribeiro, D. A. Ferreira, N. Bressan, C. S. Nunes, P. Amorim, L. Antunes, "Brain monitoring in dogs using the cerebral state index during the induction of anaesthesia via target controlled infusion of propofol," Research in Veterinary Science, 2007,

[8] F. Guarracino, F. Lapolla, C. Cariello, A. Danella, L. Doroni, R. Baldassarri, A. Boldrini, M. L. Volpe, "Target controlled infusion: TCI," Minerva Anestesiologica, vol. 71, pp 335-7, 2005.

[9] A. Absalom, M. M. R. F. Struys, "An Overview of Target Controlled Infusions and Total Intravenous Anaesthesia, Academia Press, Gent, Belgium; 2005.

[10] T. Beths, J. B. Glen, J. Reid, A. M. Monteiro, A. M. Nolan, "Evaluation and optimisation of a target-controlled infusion system for administering propofol to dogs as part of a total intravenous anaesthetic technique during dental surgery," The Veterinary Record, vol. 148, pp 198-203, 2001.

[11] S. Brás, N. Bressan, L. Ribeiro, L. Antunes, C. S. Nunes, "Pharmacokinetics and Pharmacodynamics of Propofol in Dog: KeO using the Cerebral State Index," Anesthesiology, vol. 107, pp A827, 2007.

[12] G. C. Musk, D. S. J. Pang, T. Beths, D. A. Flaherty, "Target controlled infusion of propofol in dogs evaluation of four targets for induction of anaesthesia," The Veterinary Record, vol. 157, pp 766-70, 2005.

[13] Danmeter A/S, Cerebral State Monitor, User Manual. Kildemosevej 13, DK-5000 Odense C, Denmark, 2006.

[14] Z. Kovacic and S. Bogdan, Fuzzy Controller Design Theory and Applications, Taylor \& Francis Group; 2006.

[15] K. Astrom and B. Wittenmark, Adaptive Control, Addison-Wesley Publishing Company; 1995. 